\title{
Effect of the Processing Temperature on the Degradation of Food Flavonoids: Kinetic and Calorimetric Studies on Model Solutions
}

\author{
Irina Ioannou ${ }^{1,2}$, Alexandre Kriznik ${ }^{3}$, Leila Chekir ${ }^{4}$, Mohamed Ghoul ${ }^{1}$ \\ 1. Laboratory Reactions and Process Engineering (LRGP), Lorraine University, Vandoeuvre, France \\ 2. URD Agro-Biotechnologies Industrielles (ABI)-AgroParisTech, CEBB 3 rue des Rouges Terres, F-51110 \\ Pomacle, France \\ 3. Biopôle, Lorraine University, Vandoeuvre, France \\ 4. Laboratory of Cellular and Molecular Biology, Faculty of Dental Medicine, University of Monastir, Monastir, \\ Tunisia \\ E-mail: irina.ioannou@agroparistech.fr
}

Received: 30 September 2019; Accepted: 20 October 2019; Available online: 30 November 2019

\begin{abstract}
Food flavonoids have a high antioxidant activity that make them bioactive to fight oxidation. However, when they are used in different formulations, these bioactive molecules are often the subject of at least one heat treatment step such as cooking, pasteurization. To establish the sensitivity of these molecules to heat treatment, the kinetics of degradation of 4 flavonoid extracts were evaluated under isothermal and non-isothermal conditions. The flavonoids tested belong to the aglycon (eriodictyol and mesquitol) and glycosylated (rutin, naringin) forms. The glycosylated flavonoids have a higher stability than the aglycons. Among the glycosylated flavonoids, rutin is more sensitive to heat than naringin because of the different degree of hydroxylation between these 2 compounds. For the aglycon form, the sensitivity to heat varies depending on the absence or presence of a carbonyl group at the 4-position and a hydroxyl at the 3-position. The addition of $10 \%$ of propylene glycol causes an effect protector delaying the thermal degradation of flavonoids. Activation energies are also affected by both the flavonoid structure and the heat treatment system. The differences observed between the isothermal and non-isothermal conditions can be attributed to the difference in heating intensity that can generate a difference in the composition of the solution. The evolution of antioxidant activity of flavonoid solutions after heat treatment is different depending on the structure of the flavonoid studied.
\end{abstract}

Keywords: Thermal stability; Heat processing system; Flavonoid structure; Calorimetry; Kinetic parameters.

\section{Introduction}

Flavonoids are antioxidant molecules widespread in fruit and vegetables. Recent epidemiological studies have shown that a diet of fruit and vegetables rich in flavonoids leads to a general well-being of consumers. This is attributed to biological activities of flavonoids particularly their antioxidant activity. According to TomásBarberán et al. [1], foods rich in flavonoids reduce specific diseases such as diabetes, cancer, cardiovascular or neuronal diseases. Numerous studies have dealt with the quantification of flavonoid contents and of their activities in raw food. However, the human diet is mainly based on the consumption of products having undergone heat treatment during their transformation from raw materials to finished products. The behaviour of flavonoids after processing and their incorporation into a food matrix has scarcely been studied. A review on this subject, realized by Ioannou et al. [2], revealed that food processing induces a severe degradation of flavonoids and consequently a modification of their antioxidant activities. However, the intensity of the degradation seemed to change depending on the process and its operating conditions, the composition of the food matrix and on the structure of the flavonoids. Indeed, several authors indicated that glycosylated flavonols are less sensitive to heat treatment than aglycon ones [3-5]. However, according to Murakami et al. [6], luteolin 7-O glucoside is more sensitive when heated to a temperature of $100^{\circ} \mathrm{C}$ than its aglycon form luteolin. Moreover, for a given aglycon isoflavone, the sensitivity to heat treatment is variable depending on the degree of hydroxylation [7].

The objective of this paper is to investigate the effect of the processing temperature on the flavonoid degradation and the evolution of their antioxidant activities. In first, the effect of the extraction temperature will be determined on the flavonoid content of an orange peel extract. Then, in a second time, the effect of the temperature will be determined on flavonoid model solutions to avoid interactions with food matrix. The degradation kinetics and the evolution of the antioxidant activity of 4 flavonoids belonging to aglycon and glycosylated forms will be studied (Figure 1). These solutions were submitted to heat processing under isothermal and non isothermal conditions 
under a wide range of temperatures varying from 30 to $130^{\circ} \mathrm{C}$. Indeed, the heat processing system in isothermal conditions do not take the steps of raising and lowering temperature into account. However, these steps can affect the kinetic parameters of degradation. Thus, it is also necessary to study the effect of heat processing in non isothermal conditions. The recorded profiles were used to compare the rate of degradation and activation energy. The residual antioxidant activity of the solutions was also evaluated.

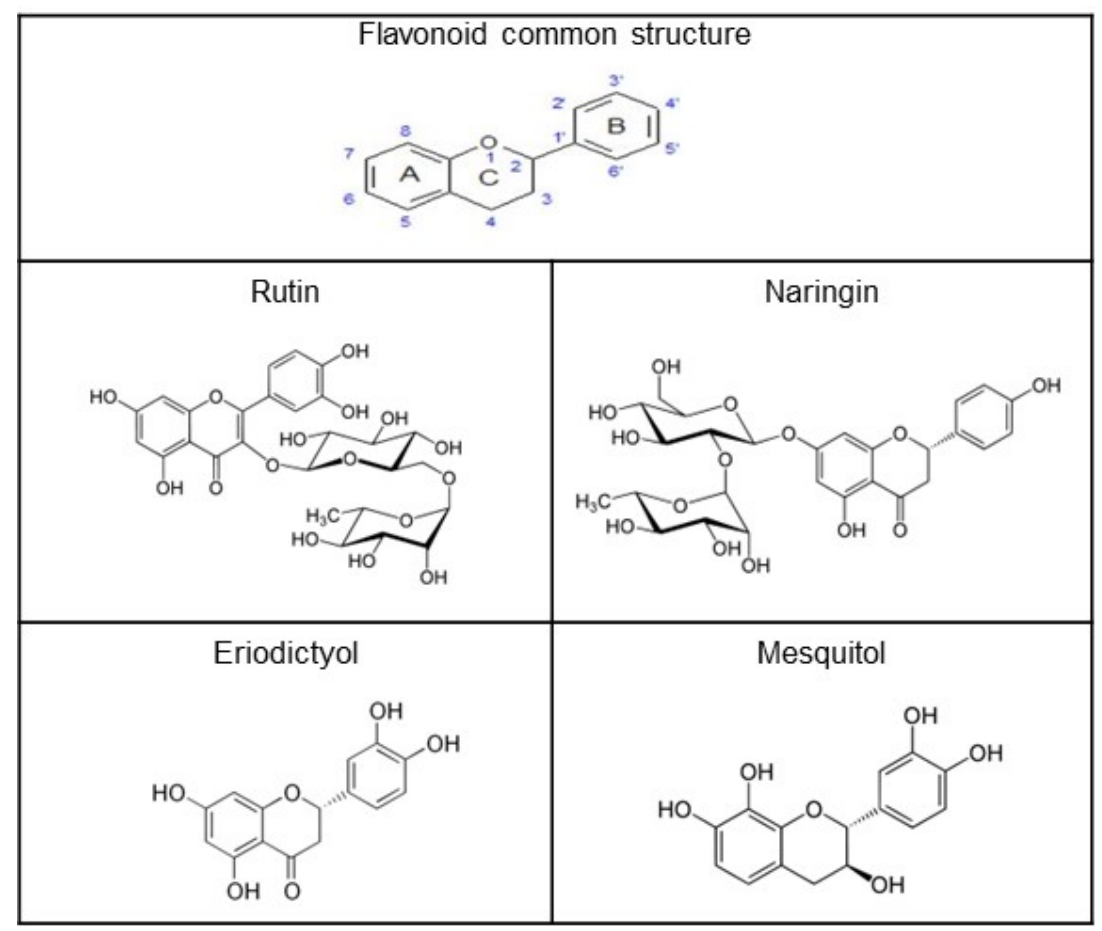

Figure 1. Structure of the 4 flavonoids studied

\section{Material and methods}

\subsection{Chemicals}

Rutin was obtained from Alfa Aesar, naringin was supplied by Sigma-Aldrich, eriodictyol was purchased from Extrasynthese. Mesquitol was extracted from a Kenya tree proposis juliflora. Structural elucidation was based on FTIR, 1H and 13C NMR, GC-MS and HPLC analyzes. Results obtained showed the obtaining of (-) mesquitol as a sole compound with a high purity [8]. ABTS (2'-azinobis (3-ethyl-benzothiazoline-6-sulfonic acid) diammonium salt) was purchased from Sigma-Aldrich Chemical. Trolox and potassium persulfate were purchased from Fluka. Ethanol and propylene glycol (PG) were obtained from VWR. Propylene glycol was obtained from Amresco. Methanol was purchased from Carlo Erba. All reagents and solvents were of analytical grade.

\subsection{Extraction of phenolic compounds from orange peel by microwaves}

A laboratory scale microwave extraction apparatus (Multiwave 3000, Austria) was used for extraction. $5 \mathrm{~g}$ of orange peel powder were extracted with $50 \mathrm{~mL}$ of $80 \%$ ethanol, 3 times. Samples were heated at $100 \mathrm{~W}$ to $400 \mathrm{~W}$ for $180 \mathrm{~s}$. In these conditions, the temperature of extraction medium is $67^{\circ} \mathrm{C}$ for $100 \mathrm{~W}, 76^{\circ} \mathrm{C}$ for $200 \mathrm{~W}, 94^{\circ} \mathrm{C}$ for $300 \mathrm{~W}$ and $108^{\circ} \mathrm{C}$ for $400 \mathrm{~W}$. the extracts were cooled to room temperature and immediately centrifuged at 8000 $\mathrm{g}$ for $10 \mathrm{~min}$ and the supernatant was filtered through a $0.2 \mu \mathrm{m}$ solvent filter. The contents in hesperidin, neohesperidin, naringin, narirutin, sinensetin, didymin, and tangeretin were quantified by HPLC.

\subsection{Flavonoid model solutions}

A glycosyl flavonol (rutin), a glycosyl flavanone (naringin), an aglycon flavanone (eriodictyol), and an aglycon flavon-3-ol (mesquitol) were chosen because (i) they belong to flavonoid classes often found in food raw materials, (ii) they are easily available and (iii) different structural elements are absent or present like enone structure (double bond in C2-C3), carbonyl group in position 4, hydroxyl in position 3, and glycosyl groupment.

Solutions of flavonoids were prepared according to their solubility: naringin $0.04 \mathrm{mM}$, rutin $0.04 \mathrm{mM}$, mesquitol $0.08 \mathrm{mM}$, and eriodictyol $0.08 \mathrm{mM}$. 2 solvents were used to prepare these solutions: $100 \%$ water and a mix of water and propylene glycol (90/10 \%). Propylene glycol was used to increase the solubility of flavonoids in water[9,10]. Moreover, it can be used as additive in food products (Commission Regulation (EC) No 1333/2008). 
For the preparation of water solutions, flavonoids were dissolved in water at a temperature of $25^{\circ} \mathrm{C}$ for one half hours in the dark using stirring $(600 \mathrm{rpm})$. For the preparation of propylene glycol model solutions, flavonoids were dissolved in propylene glycol for $10 \mathrm{~min}$, then water was added to obtain a proportion of $90 \%$ of water. The mix was agitated $(600 \mathrm{rpm})$ in the dark at a temperature of $25^{\circ} \mathrm{C}$ for a total duration of one hour and half.

\subsection{Thermal processing of flavonoid model solutions}

\subsubsection{Thermal processing under isothermal conditions}

Flavonoid solutions were heated at different temperatures (30,50, 70, 90, 110, and $130^{\circ} \mathrm{C}$ ) for $2 \mathrm{~h}$ in an oilbased bath (Hubert, W8518D). Samples were taken every $15 \mathrm{~min}$ and cooled to a temperature of $30^{\circ} \mathrm{C}$. Then, samples were analyzed by HPLC. Residual concentrations were plotted according to heating time.

Different models were tested to fit the degradation kinetics of the flavonoids, however, only the model of order 1 allows coefficients of determination superior to 0.9 to be obtained. Thus, Equation (1) was used to calculate the constant rates of the degradation reactions $(k)$.

$$
\ln \left(C / C_{0}\right)=-k t
$$

where $\mathrm{C}_{0}$ is the initial flavonoid content, and $\mathrm{C}$ the flavonoid content after $\mathrm{t}$ minutes of heating at a given temperature, $\mathrm{k}$ is the reaction rate constant $\left(\mathrm{min}^{-1}\right)$.

The Arrhenius model (Equation (2)) was used for activation energy determination:

$$
\ln k=\ln k_{O}-\frac{E a}{R T}
$$

\subsubsection{Thermal processing under non-isothermal conditions}

A VP-DSC microcalorimeter (VPDSC expanded range microcalorimeter, Microcal) was used to perform the microcalorimetry analyzes. Model solutions are degassed over $8 \mathrm{~min}$ (Thermovacsample degassing and thermostat, Microcal Inc., vacuum), then, $1 \mathrm{~mL}$ was injected into the microcalorimeter cell. Samples were scanned by temperature ramping from 30 to $130^{\circ} \mathrm{C}$ at a scanning rate of $4^{\circ} \mathrm{C} / \mathrm{h}$. Analysis of the data was performed by the Origin 7.0 software package (Microcal), and heat capacities $(\triangle C p)$ were obtained for every temperature. Enthalpies $(\Delta H)$ of the degradation reactions were calculated after subtraction of the sample from the reference.

Constant rates at each temperature were determined according to Equation (3):

$$
k_{T, a v}=-\frac{\Delta A / \Delta t}{A_{a v}}
$$

where $\Delta A$ is the change in flavonoid concentration at each time interval (Equation (4)), $\Delta t$ is the total time of the microcalorimetry analysis and $A_{a v}$ is the average flavonoid concentration during each time interval (Equation (5)).

$$
\Delta A=\left(\frac{\Delta H}{H_{T}}\right) \times \Delta A_{T}
$$

where $\Delta H$ is the change in enthalpy within the time interval $\left(\mathrm{kcal}_{\mathrm{mol}}{ }^{-1}\right), H_{\mathrm{T}}$ is the total enthalpy of the degradation reaction and $\Delta A_{T}$ is the degraded amount of flavonoid measured by HPLC.

$$
A_{a v}=\left[1-\frac{\left(\frac{H_{2}+H_{1}}{2}\right)}{H_{T}}\right] \times \Delta A_{T}
$$

where, $\mathrm{H} 1$ and $\mathrm{H} 2$ are the partial decomposition enthalpies for the beginning and the end of each time interval.

The Arrhenius model was then used to calculate the activation energy.

\subsection{HPLC/DAD Analysis}

HPLC analysis was carried out using an Elite Lachrom HPLC system (VWR HITACHI) which consisted of (L2455) a diode-array detector, (L-2200) an autosampler and (L-2130) a quaternary pump coupled to a column C18 (150x4.6 mm) (Grace). The analysis method was developed in the laboratory. The mobile phases were water (phase A) and methanol (phase B). Separation was achieved by elution gradient using an initial composition of $95 \%$ water and $5 \%$ methanol, then the percentage of methanol was raised to $100 \%$ for 10 min and finally methanol dropped to $5 \%$ after $20 \mathrm{~min}$. The total running time was $30 \mathrm{~min}$. The injection volume was $50 \mu \mathrm{L}$. A flux of 1 $\mathrm{mL} / \mathrm{min}$ and a temperature of $50^{\circ} \mathrm{C}$ were used. Chromatograms were obtained at 254, 280 and $350 \mathrm{~nm}$. 


\subsection{Antioxidant activity assay}

The antioxidant activity was measured by the ABTS method described by Re et al. [11], with some modifications for use in microplates. The ABTS assays were performed with a spectrofluorimeter SAFAS (Xenius) equipped with a 96-well polystyrene plate. Scavenging free radical potentials were tested in an aqueous solution of ABTS*+. The percentage of inhibition was calculated with Equation (6).

$$
(I P \%)=100 \times\left(A_{C}-A_{S}\right) / A_{C}
$$

where $A_{C}$ and $A_{S}$ are the absorbance of the control and of the test sample, respectively.

Then TEAC was determined according to Equation 7.

$$
\mathrm{TEAC}=\frac{\mathrm{as}_{\mathrm{S}}}{\mathrm{a}_{\mathrm{T}}}
$$

where $a \mathrm{~S}$ is line slope of the percentage of inhibition plotted vs. concentration $(\mu \mathrm{mol} / \mathrm{L})$ for the sample and $a T$ is line slope of percentage of inhibition plotted $v s$. concentration $(\mu \mu \mathrm{mol} / \mathrm{L})$ for Trolox reference.

\subsection{Data Analysis}

All kinetic experiments were performed in triplicate. Averages are calculated as well as standard deviations, which are not represented in order not to overload the graphs. The Origin 7.0 software package (Microcal) and SciDAVis were used to analyze calorimetric data. The software SigmaPlot was used to model the kinetic degradations. Statistical analysis was performed on data with Ri386 3.0.2 freeware to show significant differences $(\mathrm{p}<0.5)$.

\section{Results and discussion}

First of all, the effect of the processing temperature will be observed on the degradation of flavonoids extracted from a food matrix and in a second time, kinetic and calorimetric studies will be led on flavonoid model solutions to generalize conclusions about the effect of the processing temperature on the flavonoid degradation.

\subsection{Effect of the extraction temperature on flavonoid content issue from orange peel}

An extraction by solvent accelerated by microwaves was conducted on orange peel to extract flavonoids. Different microwave powers were tested and the contents in hesperidin, neohesperidin, naringin, narirutin, sinensetin didymin, and tangeretin were determined by HPLC. The extraction temperature is proportional to the microwave power used. The different contents in flavonoids according to the extraction temperature are represented figure 2 .

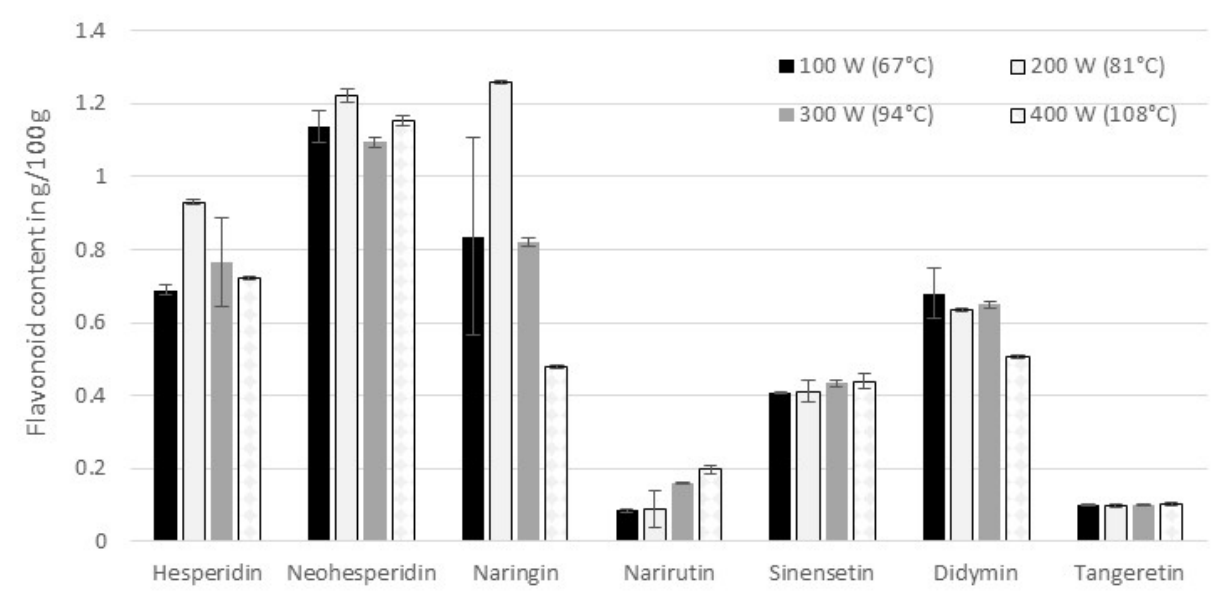

Figure 2. Flavonoid contents according to the microwave powers of the extraction (Contents of naringin, narirutin, sinensetin didymin, and tangeretin are multiplied by 10).

According to the figure 2, the optimum power leading to the highest flavonoid content is different according to the flavonoid studied. Indeed, for sinensetin and tangeretin, no effect of the power was observed whereas for the narirutin an increase of the content is noticed with an increase of the extraction power. For hesperidin, 
neohesperidin, naringin, and didymin, an optimum of the power was found. For didymin, the optimum corresponds to $300 \mathrm{~W}$ whereas for the others, the optimum is $200 \mathrm{~W}$.

An increase of the extraction temperature leads to an increase of the solubility of the flavonoid into the solvent and thus an increase of the content extracted. However, a temperature too high can lead to the degradation of the flavonoid. Thus the content measured is the difference between the flavonoids extracted and degraded. The optimum temperature can be different according to the sensitivity of the flavonoid to heat. To a better understanding of the effect of the processing temperature on the flavonoid content, it will be relevant to study flavonoid model solutions to avoid the effect of the food matrix.

\subsection{Effect of the temperature processing on flavonoid model solutions}

The degradation of rutin, naringin, eriodictyol, and mesquitol were studied under isothermal and under non isothermal procedures in water and in water with $10 \%$ of propylene glycol. Residual concentrations, antioxidant activities, constant rates, and activation energies were evaluated under these 2 operating conditions.

\subsubsection{Kinetics of degradation of flavonoids under isothermal conditions}

The degradation kinetics of flavonoids in water and in the presence of $10 \%$ of propylene glycol are presented in figures $3 \mathrm{a}$ and $3 \mathrm{~b}$ respectively. These results indicated that naringin is the least sensitive to heat treatment compared to the 3 other studied molecules. In fact, the degradation of naringin in water starts only when the temperature is at least equal to $110^{\circ} \mathrm{C}$. At this set point of temperature and even after $2 \mathrm{~h}$ of treatment more than $90 \%$ of naringin remains in solution. For the higher temperature of $130^{\circ} \mathrm{C}$ only $20 \%$ of naringin were lost. The presence of the propylene glycol does not affect the kinetic behaviour of the naringin. Unlike naringin, mesquitol is the most sensitive compound with $32 \%$ of degradation even at $50^{\circ} \mathrm{C}$ and $93 \%$ at $130^{\circ} \mathrm{C}$. For this compound, the addition of $10 \%$ of propylene glycol seems to play a protective role for temperatures below $100^{\circ} \mathrm{C}(50,70$, $90^{\circ} \mathrm{C}$ ). This effect becomes non-significant for higher temperatures.

For eriodictyol, the effect of temperature is observed from $70^{\circ} \mathrm{C}$ with $18 \%$ of loss; the concentration falls to zero after $80 \mathrm{~min}$ of incubation at $130^{\circ} \mathrm{C}$. The presence of $10 \%$ of propylene glycol in solution has also a protective effect like for the mesquitol. Indeed, for the temperatures $70,90,110$, and $130^{\circ} \mathrm{C}, 82,59,24$, and $0 \%$ of flavonoid remain in water, whereas 91, 79, 38, and $6 \%$ of eriodictyol remain in propylene glycol (10\%). The evolution of the residual concentration of rutin, exhibits 2 behaviours, a linear degradation for temperatures below $100^{\circ} \mathrm{C}$ and an exponential degradation for higher temperatures. The propylene glycol slightly decreases the effect of temperature. These results indicate that whatever the heating temperature, either the final residual concentration is higher or the degradation rate is lower in the presence of propylene glycol. So, it seems that the propylene glycol delays the flavonoid denaturation during heat treatment due to its protective effect.

The observed difference in sensitivity among the 4 flavonoids can be explained by the differences in their structures such as the presence or absence of a glycosyl group, the degree of hydroxylation and the number of double bonds. Our results indicated that glycosylated flavonoids (naringin, rutin) are less sensitive to heat than the aglycons (eriodictyol, mesquitol). These results are in agreement with the data reported by Makris et al. and Buchner et al. [3,5]. These authors showed that rutin is less sensitive to heat than its aglycon equivalent quercetin. Our results also indicated that rutin is more sensitive to heat treatment than naringin even though these 2 compounds are both glycosylated. This behaviour can be attributed to the degree of hydroxylation. The most hydroxylated compounds are more affected by heat treatment.

Ungar et al. [7] observed a similar phenomenon during their studies of 2 isoflavones having different degrees of hydroxylation. For aglycon compounds, sensitivity is not only related to the degree of hydroxylation, but also to the presence of a carbonyl group in position 4 and the presence of a hydroxyl in position 3. Indeed, the flavonoids having a hydroxyl in position 3 are highly affected by temperature, oxygen, and light [12]. This can explain the high sensitivity of mesquitol compared to eriodictyol.

\subsubsection{Kinetic parameters of the flavonoid degradation}

As is indicated in paragraph 2.3 the best correlation of the degradation kinetics was obtained by a model of first order. Thus, constant rates of degradation are determined by plotting $\ln (\mathrm{C} / \mathrm{Co})$ according to heating time. Arrhenius law is then applied to find the activation energy of degradation for each flavonoid. Table 1 shows the constant rates and the activation energies after a heating of 2 hours. Naringin in $100 \%$ water shows a starting degradation at $110^{\circ} \mathrm{C}$, its degradation constant rate at $130^{\circ} \mathrm{C}$ is low $\left(0.0019 \mathrm{~min}^{-1}\right)$. The denaturation of the rutin and the eriodictyol in $100 \%$ water is observed at a temperature of $70^{\circ} \mathrm{C}$. Rutin shows 2 profiles of degradation: slowly below $100^{\circ} \mathrm{C}\left(0.0037 \mathrm{~min}^{-1}\right.$ at $\left.90^{\circ} \mathrm{C}\right)$ and more rapidly above $100^{\circ} \mathrm{C}\left(0.0316 \mathrm{~min}^{-1}\right.$ at $\left.110^{\circ} \mathrm{C}\right)$. Mesquitol is the most sensitive, its denaturation appears at a temperature of $50^{\circ} \mathrm{C}$. However at $130^{\circ} \mathrm{C}$, the mesquitol degradation constant rate $\left(0.0236 \mathrm{~min}^{-1}\right)$ is lower than the rutin degradation constant rate $\left(0.0444 \mathrm{~min}^{-1}\right)$. 


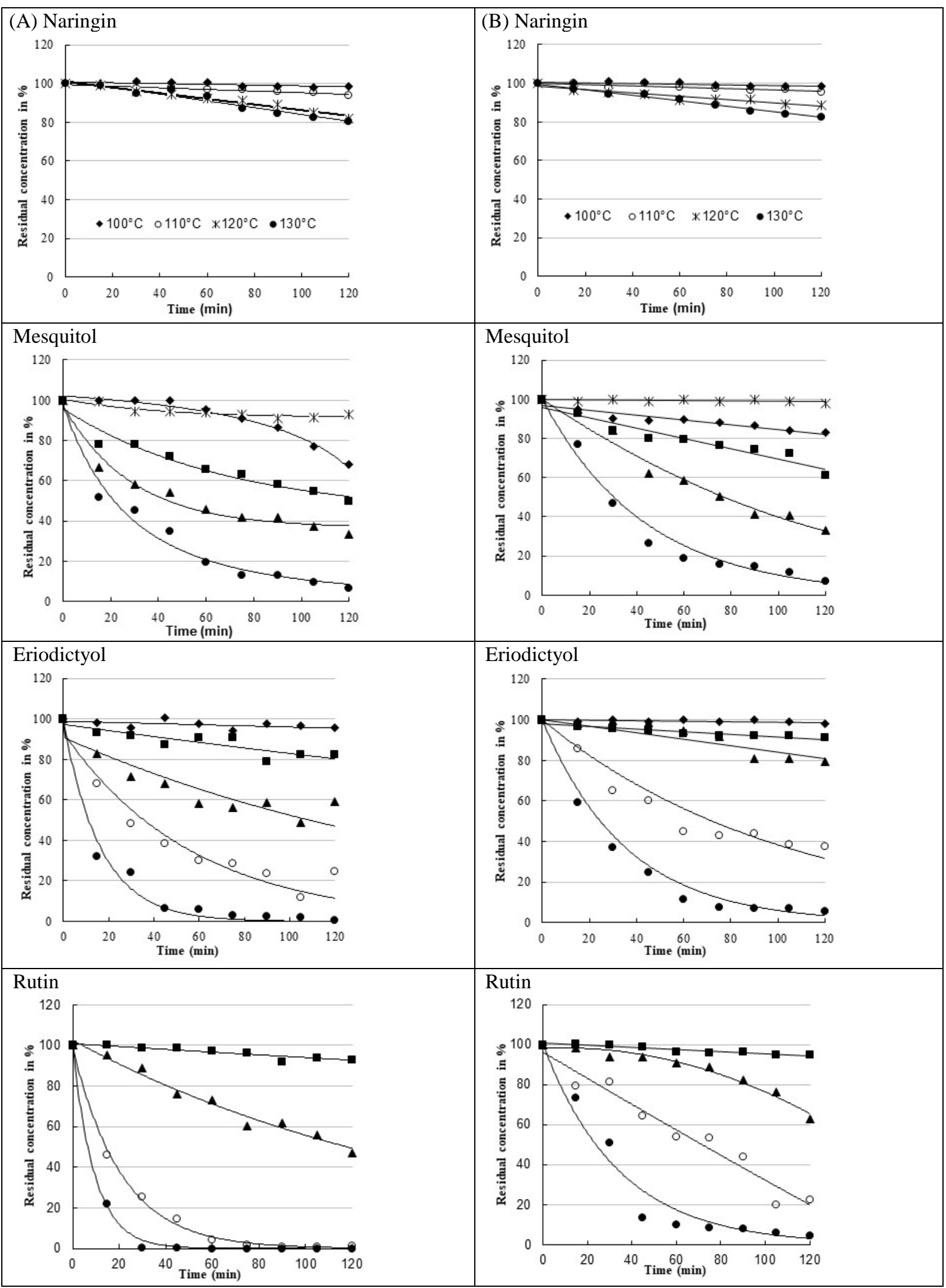

Figure 3. Degradation kinetics of flavonoids at different temperatures over $2 \mathrm{~h}\left(\times 30^{\circ} \mathrm{C} \bullet 50^{\circ} \mathrm{C} \boldsymbol{\bullet} 70^{\circ} \mathrm{C} \boldsymbol{\Delta} 90^{\circ} \mathrm{C}\right.$ $\mathrm{O} 110^{\circ} \mathrm{C} \bullet 130^{\circ} \mathrm{C}$ )

These phenomena can be explained by the difference of flavonoid structure. In fact, naringin and rutin which are glycosylated flavonoids need high energies to degrade: $78 \mathrm{~kJ} / \mathrm{mol}$ for rutin and $101 \mathrm{~kJ} / \mathrm{mol}$ for naringin. This high energy can be attributed to the presence of the glycosylated groups. For the other flavonoids, activation 
energies are weaker. It is almost $68 \mathrm{~kJ} / \mathrm{mol}$ for eriodictyol due to the presence of the carbonyl group in C4 and 33 $\mathrm{kJ} / \mathrm{mol}$ for mesquitol. Thus, it appears clearly that flavonoids with high activation energy are characterized by a high starting temperature of degradation.

All the constant rates are significantly lower in propylene glycol than in $100 \%$ water, except for naringin $\left(110^{\circ} \mathrm{C}\right)$ and for mesquitol $\left(130^{\circ} \mathrm{C}\right)$. However, the activation energies are of the same order of magnitude for the 2 solvents. This phenomenon can be explained by the fact that the activation energy represents the energy necessary to break covalent bonds. The addition of propylene glycol in solution increases only the number of non-covalent bonds with flavonoids (Van der Waals interaction, hydrogen bonds) without affecting the number of covalent bonds.

Table 1. Kinetic parameters of rutin, naringin, eriodictyol and mesquitol under isothermal heating $\left(\mathbf{k . 1 0} \mathbf{1 0}^{\mathbf{3}}\left(\mathrm{min}^{-1}\right)\right)$

\begin{tabular}{|c|c|c|c|c|c|c|c|c|c|}
\hline & & \multicolumn{2}{|l|}{ Naringin } & \multicolumn{2}{|l|}{ Eriodictyol } & \multicolumn{2}{|l|}{ Mesquitol } & \multicolumn{2}{|l|}{ Rutin } \\
\hline & & $\begin{array}{l}\text { In } 100 \% \\
\text { water }\end{array}$ & $\begin{array}{l}\text { In } 10 \% \\
\text { PG }\end{array}$ & $\begin{array}{l}\text { In } 100 \% \\
\text { water }\end{array}$ & $\begin{array}{l}\text { In } 10 \% \\
\text { PG }\end{array}$ & $\begin{array}{l}\text { In } 100 \\
\text { water }\end{array}$ & $\begin{array}{l}\text { In } 10 \\
\text { PG }\end{array}$ & $\begin{array}{l}\text { In } 100 \% \\
\text { water }\end{array}$ & $\begin{array}{ll}\text { In } 10 \% \\
\text { PG }\end{array}$ \\
\hline \multirow[t]{6}{*}{$\mathbf{T}\left({ }^{\circ} \mathrm{C}\right)$} & 50 & nd & nd & nd & nd & $2 \pm 0.1$ & $1.7 \pm 0.1$ & nd & nd \\
\hline & 70 & nd & nd & $1.7 \pm 0.1$ & $0.9 \pm 0.1$ & $6.1 \pm 0.2$ & $3.7 \pm 0.2$ & $1 \pm 0.1$ & 0.5 \\
\hline & 90 & nd & nd & $7.3 \pm 0.2$ & $1.7 \pm 0.1$ & $10.4 \pm 0.2$ & $9.1 \pm 0.2$ & $3.7 \pm 0.1$ & $2.7 \pm 0.1$ \\
\hline & 110 & 0.4 & $0.3 \pm 0.1$ & $18.7 \pm 0.4$ & $9.8 \pm 0.2$ & ni & ni & $31.6 \pm 0.7$ & $11.6 \pm 0.3$ \\
\hline & 120 & $1.6 \pm 0.1$ & $1.1 \pm 0.1$ & ni & ni & - & - & - & - \\
\hline & 130 & $1.9 \pm 0.1$ & $1.6 \pm 0.1$ & $46.9 \pm 1.1$ & $28.9 \pm 0.7$ & $23.6 \pm 0.6$ & $23.2 \pm 0.5$ & $44.4 \pm 1.1$ & $29.1 \pm 0.7$ \\
\hline & (1) & $101 \pm 10$ & $108 \pm 10$ & $68 \pm 2$ & $69 \pm 1$ & $33 \pm 2$ & $36 \pm 2$ & $78 \pm 2$ & \\
\hline
\end{tabular}

* nd: not degraded ; ni: not investigated

\subsection{Thermal treatment under non-isothermal conditions}

To characterize the degradation of the 4 flavonoids presented previously, the calorimetric technique was used to induce a heat treatment from $30^{\circ} \mathrm{C}$ to $125^{\circ} \mathrm{C}$ with an increment of $4^{\circ} \mathrm{C}$ per hour. The heat generated by the degradation of each compound was recorded throughout along the treatment. The profiles obtained were used to identify the temperature from which flavonoid degradation begins, to calculate the enthalpies and the constant rates of the degradation reactions. Thus, activation energies are determined by the use of Arrhenius law.

\subsubsection{Scans obtained by microcalorimetry}

In figure 4, the evolution of heat capacity (Cp) according to time is plotted for each molecule in water and in propylene glycol (10 \%). The naringin scan indicates that this molecule remains stable between 30 and $130^{\circ} \mathrm{C}$.

For rutin, mesquitol, and eriodictyol, their scans exhibit a large exothermic peak which indicates that a degradation reaction occurs under these conditions. The degradation of rutin starts at a temperature of $67^{\circ} \mathrm{C}$ and $82^{\circ} \mathrm{C}$ respectively in water and with $10 \%$ propylene glycol. The degradation reactions reach their maximum at $98^{\circ} \mathrm{C}$ in $100 \%$ water and $115^{\circ} \mathrm{C}$ in $10 \%$ propylene glycol. The 2 peaks are similar with a shift of $15^{\circ} \mathrm{C}$.

For eriodictyol, the reactions start at a temperature of $60^{\circ} \mathrm{C}$ for the 2 solutions. However, like for rutin, the maximum rate of degradation is reached at $105^{\circ} \mathrm{C}$ in the presence of water and $111^{\circ} \mathrm{C}$ with $10 \%$ of propylene glycol. Mesquitol is the most sensitive molecule whose degradation starts at $50^{\circ} \mathrm{C}$ and the maxima are reached at a temperature of $94^{\circ} \mathrm{C}$ for the both solutions tested. These results indicate that the sensitivity to this system of heat processing is variable depending on the structure of the molecule, as discussed above.

\subsubsection{Kinetic parameter determination}

At the end of each thermal analysis, the final flavonoid concentration was determined by HPLC. The measured enthalpies were normalized for the actual amount of flavonoid being degraded. The constant rates of degradation were determined in a range of temperature in which an exponential increase for degradation rate was observed. The calculated degradation constant rates were used to plot lnk vs $1 / \mathrm{T}$ and the activation energies were calculated according to Arrhenius equation. Good determination coefficients were obtained for all the conditions ( $\left.\mathrm{R}^{2}>0.9\right)$, figure 5 shows an example of determination.

The kinetic parameters calculated (residual concentrations, $\Delta \mathrm{H}$ and activation energies) are presented in table 2. These results indicate that the flavonoids in solution with $10 \%$ of propylene glycol are less sensitive to heat treatment than in water. In fact, the residual concentrations are respectively 13,13 , and $7 \%$ for, respectively, rutin, eriodictyol, and mesquitol against 1, 0, and 3\% in water. These results are in accordance with those observed under isothermal conditions. So, it can be concluded that propylene glycol has a protective effect on flavonoids during heat treatment.

The areas of the exothermal peaks were used to calculate $\Delta \mathrm{H}$. For mesquitol, the corresponding enthalpies are respectively 663 and $528 \mathrm{~kJ} / \mathrm{mol}$ in water and in $10 \%$ propylene glycol. For eriodictyol, the $\Delta \mathrm{H}$ are comparable for the 2 solutions and equal respectively to 1022 and $1079 \mathrm{~kJ}_{\text {. mol}}{ }^{-1}$. In the case of rutin, the $\Delta \mathrm{H}$ are respectively 
1085 and $893 \mathrm{~kJ} / \mathrm{mol}$. These values indicate that, whatever the solution used, the lowest enthalpy of degradation is reached in the presence of mesquitol. For rutin and eriodictyol, the $\Delta \mathrm{H}$ present similar magnitude except for rutin in $10 \%$ propylene glycol. The activation energies were of the same order of magnitude whatever the solvent used; statistical analysis shows non-significant differences between the different values obtained.

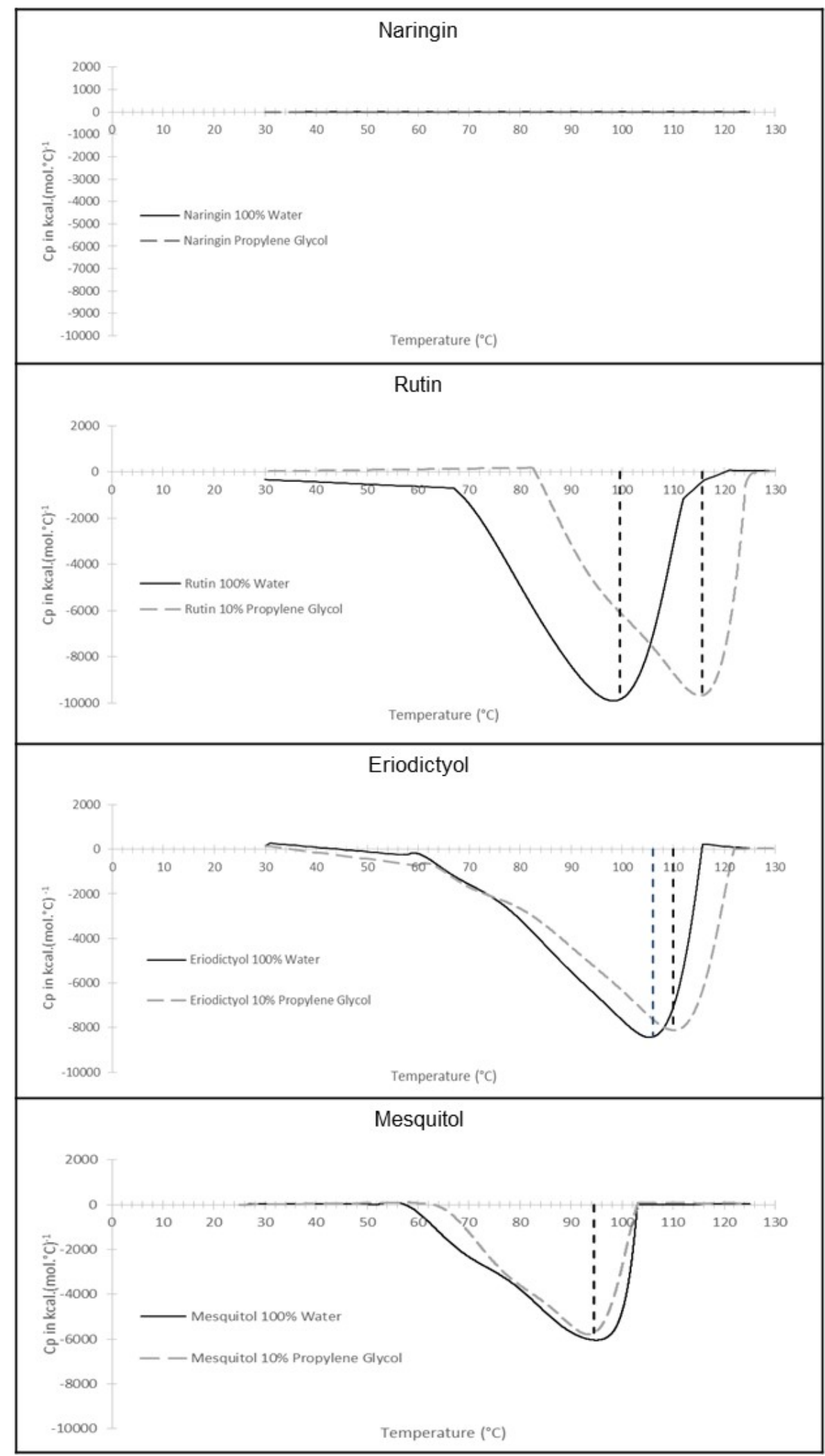

Figure 4. Scans of naringin, rutin, eriodictyol and mesquitol during microcalorimetric stability tests 


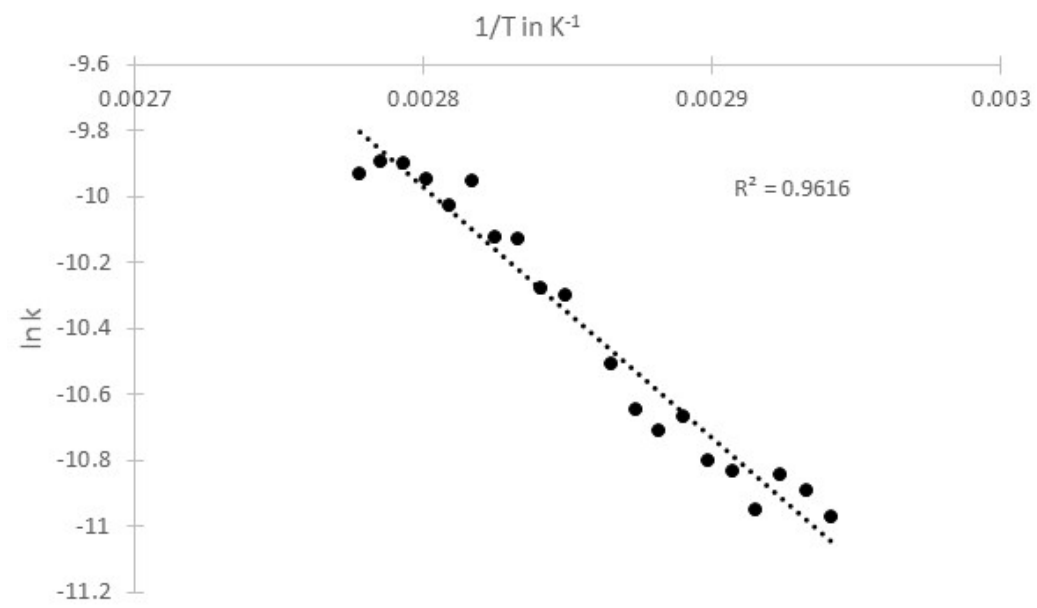

Figure 5. Determination of activation energy according to Arrhenius equation between 67 and $87^{\circ} \mathrm{C}$ for degradation of eriodictyol in water by microcalorimetry

Table 2. Enthalpy change and activation energies of flavonoid degradation as determined by microcalorimetry

\begin{tabular}{lllllll}
\hline & \multicolumn{5}{c}{ Water $100 \%$} & PG $10 \%$ \\
\cline { 2 - 7 } & RC $\%$ & $\Delta \mathrm{H}(\mathrm{kJ} / \mathrm{mol})$ & $\mathrm{Ea}(\mathrm{kJ} / \mathrm{mol})$ & $\mathrm{RC} \%$ & $\Delta \mathrm{H}(\mathrm{kJ} / \mathrm{mol})$ & $\mathrm{Ea}(\mathrm{kJ} / \mathrm{mol})$ \\
\hline Rutin & $1 \pm 2$ & $1085 \pm 40$ & $61 \pm 5$ & $13 \pm 2$ & $893 \pm 92$ & $66 \pm 4$ \\
Eriodictyol & $0 \pm 2$ & $1022 \pm 38$ & $57 \pm 10$ & $13 \pm 2$ & $1079 \pm 39$ & $62 \pm 12$ \\
Mesquitol & $3 \pm 2$ & $663 \pm 24$ & $58 \pm 3$ & $7 \pm 2$ & $528 \pm 20$ & $55 \pm 2$ \\
\hline
\end{tabular}

RC: residual concentration of flavonoid

\subsection{Antioxidant activity}

The denaturation under heat treatment of flavonoids can affect their antioxidant activities. Thus, the evolution of this activity was assessed before and after heat treatment. The antioxidant activity of each flavonoid was measured using the ABTS assay. The antioxidant activity of flavonoids before heat treatment (control) were equal to $2.66 \pm 0.34 \mathrm{mmol} / \mathrm{L}$ for rutin, $1.34 \pm 0.04 \mathrm{mmol} / \mathrm{L}$ for mesquitol, $1.26 \pm 0.09 \mathrm{mmol} / \mathrm{L}$ for eriodictyol, and $0.30 \pm 0.09 \mathrm{mmol} / \mathrm{L}$ for naringin. These values are in accordance with the results obtained by Heim et al. [13]. The highest antioxidant activity was reached with molecules having an enone structure or a catechol structure. These 2 elements are missing in the naringin structure (Figure 1) which explains its low antioxidant activity. Only rutin possesses an enone structure combined with a carbonyl group in position 4, which gives this flavonoid the highest antioxidant activity among the 4 flavonoids studied. The antioxidant activities after heat treatment are shown in figure 6. These profiles are expressed as a percentage of the measured activities compared to initial antioxidant activity. This allows a distinction between antioxidant activity due to the residual flavonoid (represent in light grey) and antioxidant activity due to the by-products of the degradation (represented in dark grey).

The results obtained indicate that despite its low degradation, the heated solution of naringin, exhibits a high antioxidant activity with an increase of up to $140 \%$ at the temperature of $130^{\circ} \mathrm{C}$. No significant differences in the antioxidant activities were observed between samples in water or in propylene glycol (10 \%). For eriodictyol in water, a significant increase of antioxidant activity was observed at a temperature of $90^{\circ} \mathrm{C}$. This increase could be attributed to the antioxidant activities of by-products (dark grey in figure 5). However, at a temperature of $130^{\circ} \mathrm{C}$, this antioxidant activity decreases. This could be explained by the fact that the degradation is sequential leading to end by-products with low antioxidant activities. For this compound, the addition of propylene glycol (10 \%) allows a constant antioxidant activity to be maintained during the heat treatment except at $130^{\circ} \mathrm{C}$ where it decreases slightly.

This behaviour can be attributed to the protective effect of the propylene glycol. For mesquitol, no significant differences appear in antioxidant activity between the control and after a thermal treatment whatever the temperature applied and whatever the solvent used. The by-products generated from mesquitol seemed to have an antioxidant activity similar to the native flavonoid.

For rutin, the antioxidant activity remains constant up to a heat treatment of $90^{\circ} \mathrm{C}$. Then, it decreases at higher temperature $\left(110\right.$ and $\left.130^{\circ} \mathrm{C}\right)$. However, the decrease is lower in propylene glycol than in water. This behaviour can be explained by the fact that the products of the degradation have an antioxidant activity comparable to rutin for a temperature below $90^{\circ} \mathrm{C}$. The decrease of the activities could be attributed to further degradation of the intermediary by-products. The difference of the antioxidant activity between samples in water and in propylene glycol can be due, as explained previously, to the protective effect of the propylene glycol. 


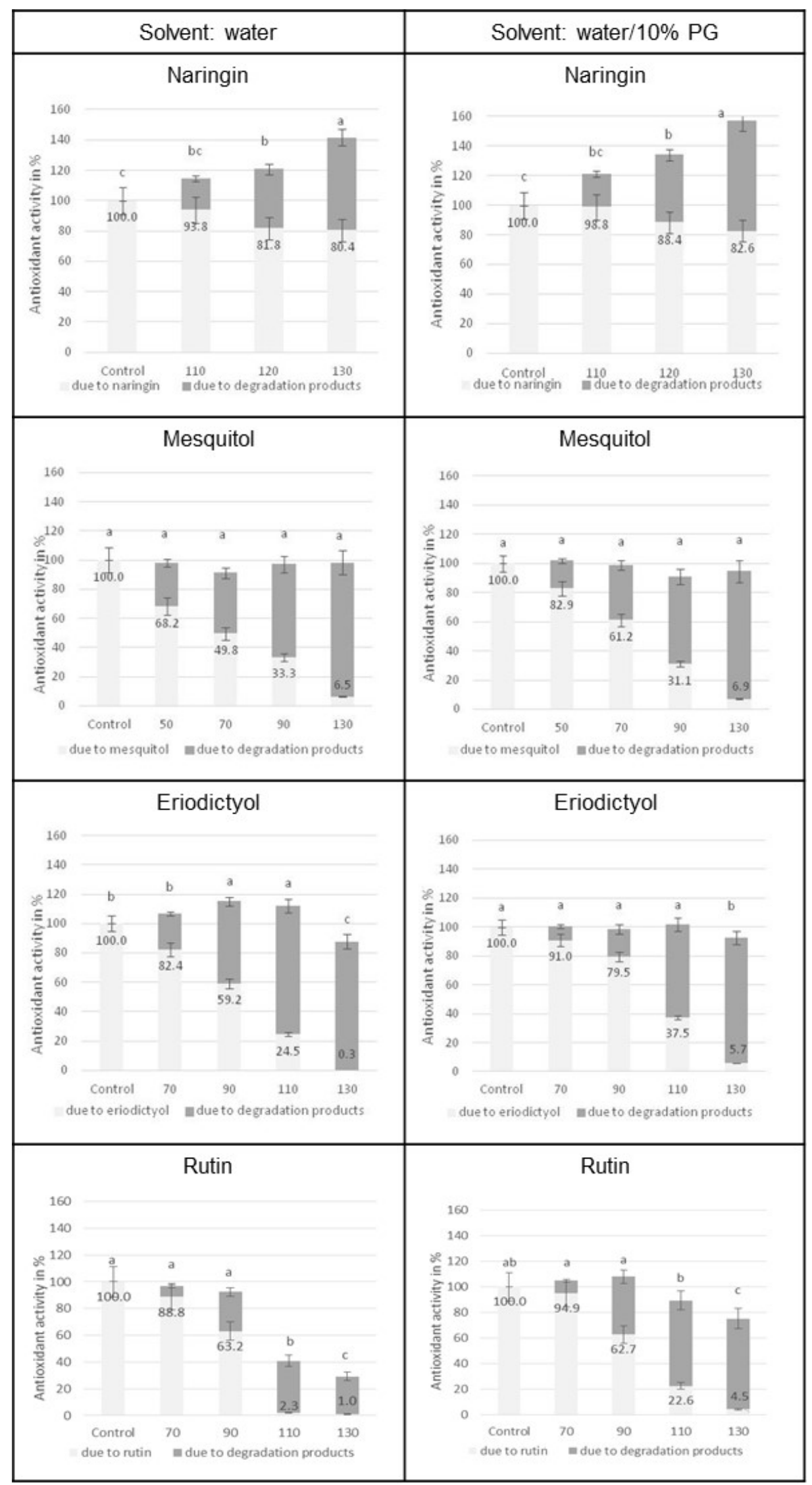

Figure 6. Evolution of the antioxidant activity of flavonoids after thermal treatment (Residual concentration of flavonoid is indicated on the charts) 


\subsection{Comparison of the 2 approaches}

The comparison of the results obtained does not indicate any difference in sensitivity between flavonoids in relation to the heat processing system. Both methods lead to similar classification from the most sensitive to the least sensitive flavonoid: mesquitol, eriodictyol, rutin, and naringin. This classification is mainly related to the structure of the flavonoids. Glycosylated flavonoids are the least sensitive as observed for rutin and naringin. These results are in accordance with literature data $[5,14]$. Aglycon flavonoids such as mesquitol and eriodictyol are more sensitive to heat treatment than glycosylated ones. However, a difference in sensitivity was also noticed within the 2 groups. Among the glycosylated flavonoids, rutin is more sensitive to heat than naringin. As was explained before, this difference is due the different degree of hydroxylation between the 2 compounds. Among the aglycon form the sensitivity to heat is variable, depending on the absence or presence of a carbonyl group on C4 and a hydroxyl in position 3.

The presence of propylene glycol does not affect the sensitivity of flavonoids but allows the delay of the effect of heat treatment. This protective effect is certainly due to an increase of non-covalent bonds in solution which gives more stability to flavonoids. However, for a given compound, the activation energy remains constant whatever the solvent used because as mentioned previously, the presence of propylene glycol creates non-covalent bonds which does not affect the activation energy.

The comparison of the activation energies under isothermal and under non isothermal conditions shows significant differences for rutin and mesquitol. In the case of eriodictyol, the activation energies are of the same order of magnitude. For rutin, a decrease of the activation energy under non isothermal conditions from $78 \pm 2$ to $61 \pm 5 \mathrm{~kJ} / \mathrm{mol}$ was observed, whereas for the mesquitol an increase from $33 \pm 2$ to $58 \pm 3 \mathrm{~kJ} / \mathrm{mol}$ was noticed.

Similar behaviour was observed during heat treatment of anthocyanin [15-17]. These authors showed that the energy of activation increases with temperature. This could be due to the coexistence in solution of several chemical species: the native flavonoids and their by-products. According to Brouillard [18], the modification of the composition of the medium affects the energy of activation.

The variation in the composition of the solutions studied leads also to a variation of kinetic parameters between isothermal and non-isothermal treatments. These differences can be attributed to the difference in the mode of treatment. In fact, in isothermal mode, the set point desired between 30 and $130^{\circ} \mathrm{C}$ was reached quickly and then the temperature was kept constant for 2 hours, whereas in non-isothermal treatment the temperature rose from 30 to $130^{\circ} \mathrm{C}$ with an increment of $4^{\circ} \mathrm{C}$ per hour, which corresponds to equivalent exposure of 24 h to $78^{\circ} \mathrm{C}$. During this long time, different linkages can take place between the solvent and the flavonoids affecting the kinetic parameters. In fact, during the storage of flavonoids, different reactions between the solvent and the flavonoids can occur particularly for flavonoids with a hydroxyl in position 3 [19].

\section{Conclusions}

To study the effect of the processing temperature, solution models of flavonoids should be used to avoid the effect of the food matrix with different chemical structure. Indeed, we noticed that the behaviour of flavonoids during by microwaves is different according to the molecule. Thus, 4 flavonoids (naringin, eriodictyol, mesquitol, and rutin) with different structures were heated under isothermal and non-isothermal conditions. The results obtained showed that the flavonoid degradation depends on the heating temperature and on the structure of the molecule. Degradation constant rates of flavonoids increase with temperature. Naringin is the least sensitive flavonoid followed by rutin, eriodictyol, and mesquitol whatever the heating conditions. The activation energies vary according to the mode of heating (isothermal or non-isothermal conditions). Activation energy increases for mesquitol, decreases for rutin and remains constant for eriodictyol. The addition of propylene glycol in solution enhances the solubility of the flavonoid and ensures a protective effect for the flavonoid against the heat treatment. Despite the degradation of flavonoids, the antioxidant activity is not lost. It seems that the degraded products could have an antioxidant activity equal or sometimes superior to native flavonoids.

\section{References}

[1] Tomás-Barberán FA, Ferreres F, Gil MI. Studies in natural products chemistry. Elsevier; 2000.

[2] Ioannou I, Hafsa I, Hamdi S, Charbonnel C, Ghoul M. Review of the effects of food processing and formulation on flavonol and anthocyanin behaviour. Journal of Food Engineering. 2012;111(2):208-217.

[3] Makris DP, Rossiter JT. Heat-induced, metal-catalyzed oxidative degradation of quercetin and rutin (quercetin 3-O-rhamnosylglucoside) in aqueous model. Journal of Agricultural and Food Chemistry. 2000;48:3830-3838.

[4] Da Costa EM, Barbosa Filho JM, Do Nascimento TG, Macedo RO. Thermal characterization of the quercetin and rutin flavonoids. Thermochimica Acta. 2019; 392-393:79-84. 
[5] Buchner N, Krumbein A, Rhon S, Kroh LW. Effect of thermal processing on the flavonols rutin and quercetin. Rapid Communications in Mass Spectrometry. 2006;20:3229-3235.

[6] Murakami M, Yamaguchi T, Takamura H, Matoba T. Effects of thermal treatment on radical-scavenging activity of single and mixed polyphenolic compounds. Food Chemistry and Toxicology. 2004;69: FCT7FCT10.

[7] Ungar Y, Osundahunsi OF, Shimoni Y. Thermal stability of genistein and daidzein and its effect on their antioxidant activity. Journal of Agricultural and Food Chemistry. 2003; 51:4394-4399.

[8] Sirmah P, Dumarcay S, Masson E, Gerardin P. Unusual amount of $(-)$ mesquitol from the heartwood of Prosopis juliflora. Natural Product Research. 2009;23(2):183-189.

[9] Shakeel F, Alshehri S, Ibrahim MA, Altamimi MA, Moshin K, Alanazi FK, Alsarra IA. Solubility and thermodynamic parameters of apigenin in different neat solvents at different temperatures. Journal of Molecular Liquids. 2017;234:73-80.

[10] Manconi M, Mariongu F, Manca ML, Caddeo C, Sarais G, Cencetti C, Pucci L, Longo V, Bacchetta G, Fadda AM. Nanoincorporation of bioactive compounds from red grape pomaces: In vitro and ex vivo evaluation of antioxidant activity. International Journal of Pharmaceutics. 2017;523:159.

[11] Re R, Pellegrini N, Proteggente A, Yang M, Rice-Evans C. Antioxidant activity applying an improved ABTS radical cation decolorization assay. Free Radical Biology \& Medicine. 1999;26(9-10):1231-1237.

[12] Smith GJ, Thomsen SJ, Markham KR, Andary C, Cardon D. The photostabilities of naturally occurring 5hydroxyflavones, flavonols, their glycosides and their aluminium complexes. Journal of Photochemistry and Photobiology A: Chemistry. 2000;136:87-91.

[13] Heim KE, Tagliaferro AR, Bobilya DJ. Flavonoid antioxidants: chemistry, metabolism and structure-activity relationships. The Journal of Nutritional Biochemistry. 2002;13:572-584.

[14] Rohn S, Buchner N, Driemel G, Rauser M, Kroh LW. Thermal degradation of onion quercetin glucosides under roasting conditions. Journal of Agricultural and Food Chemistry. 2007;55:1568-1573.

[15] Kirca A, Cemeroglu B. Degradation kinetics of anthocyanins in blood orange juice and concentrate. Food Chemistry. 2003;81(4):583-587.

[16] Harbourne N, Jacquier JC, Morgan DJ, Lyng JG. Determination of the degradation kinetics of anthocyanins in a model juice system using isothermal and non-isothermal methods. Food Chemistry. 2008;111(1):204208.

[17] Jiménez N, Bohuon P, Lima J, Dornier M, Vaillant F, Pérez AM. Kinetics of anthocyanin degradation and browning in reconstituted blackberry juice treated at high temperatures (100-180 $\left.{ }^{\circ} \mathrm{C}\right)$. Journal of Agricultural and Food Chemistry. 2010;58(4):2314-2322.

[18] Brouillard R. Anthocyanins as food colors. Academic Press; 1982.

[19] Dall'Acqua S, Miolo G, Innocenti G, Cafieri S. The photodegradation of quercetin: relation to oxidation. Molecules. 2012; 17: 8898-8907.

(C) 2019 by the author(s). This work is licensed under a Creative Commons Attribution 4.0 International License (http://creativecommons.org/licenses/by/4.0/). Authors retain copyright of their work, with first publication rights granted to Tech Reviews Ltd. 